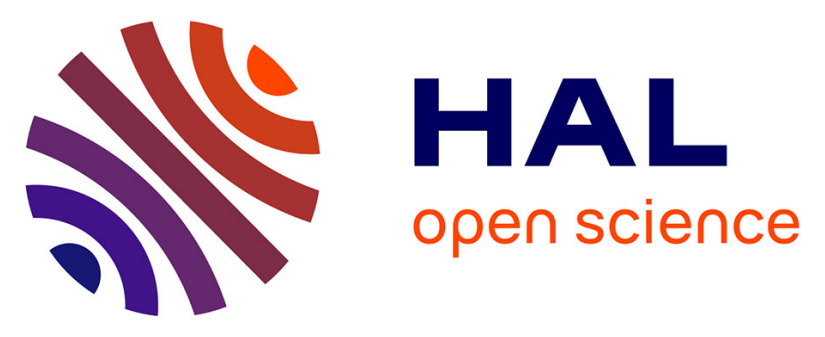

\title{
Cochlear Implantation and Other Treatments in Single-Sided Deafness and Asymmetric Hearing Loss: Results of a National Multicenter Study Including a Randomized Controlled Trial
}

Mathieu Marx, Isabelle Mosnier, Frederic Venail, Michel Mondain, Alain Uziel, David Bakhos, Emmanuel Lescanne, Yann N'guyen, Daniele Bernardeschi, Olivier Sterkers, et al.

\section{- To cite this version:}

Mathieu Marx, Isabelle Mosnier, Frederic Venail, Michel Mondain, Alain Uziel, et al.. Cochlear Implantation and Other Treatments in Single-Sided Deafness and Asymmetric Hearing Loss: Results of a National Multicenter Study Including a Randomized Controlled Trial. Audiology and Neurotology, 2021, 26 (6), pp.414 - 424. 10.1159/000514085 . hal-03460934

\section{HAL Id: hal-03460934 \\ https: / hal.sorbonne-universite.fr/hal-03460934}

Submitted on 1 Dec 2021

HAL is a multi-disciplinary open access archive for the deposit and dissemination of scientific research documents, whether they are published or not. The documents may come from teaching and research institutions in France or abroad, or from public or private research centers.
L'archive ouverte pluridisciplinaire HAL, est destinée au dépôt et à la diffusion de documents scientifiques de niveau recherche, publiés ou non, émanant des établissements d'enseignement et de recherche français ou étrangers, des laboratoires publics ou privés.

\section{(ㄷ)(1) $\$$}

Distributed under a Creative Commons Attribution - NonCommerciall 4.0 International 


\title{
Cochlear Implantation and Other Treatments in Single-Sided Deafness and Asymmetric Hearing Loss: Results of a National Multicenter Study Including a Randomized Controlled Trial
}

\author{
Mathieu Marx ${ }^{a, b}$ Isabelle Mosnier ${ }^{c}$ Frederic Venail ${ }^{d}$ Michel Mondain $^{d}$
}

Alain Uziel ${ }^{d}$ David Bakhos ${ }^{e}$ Emmanuel Lescanne ${ }^{e}$ Yann N'Guyen ${ }^{c}$

Daniele Bernardeschic ${ }^{c}$ Olivier Sterkers ${ }^{c}$ Olivier Deguine ${ }^{a, b}$ Benoît Lepage ${ }^{f}$

Benoit Godeyg Sébastien Schmerber ${ }^{h}$ Nicolas-Xavier Bonne ${ }^{\mathrm{j}}$

Christophe Vincent ${ }^{i}$ Bernard Fraysse ${ }^{a}$

\begin{abstract}
aService d'Otologie, Otoneurologie et ORL pédiatrique, Hôpital Pierre-Paul Riquet, CHU Purpan, Toulouse, France;
bBain \& Cognition Research Centre, UMR 5549, Université Toulouse III, Toulouse, France; 'AP-HP6, GHU

Pitié-Salpêtrière, Service ORL, Unité Fonctionnelle Implants Auditifs et explorations fonctionnelles, Paris, France;

dService d'ORL, Hôpital Gui de Chauliac, CHU, Montpellier, France; 'Service d'ORL, Hôpital Bretonneau, CHU Tours,

Tours, France; fUnité de Soutien Méthodologique à la Recherche, Faculté de Médecine, Université de Toulouse, Toulouse, France; '9Service d'ORL, Hôpital Pontchaillou, CHU, Rennes, France; 'hervice d'ORL, CHU, Grenoble, France; 'Service d'ORL, Hôpital Salengro, CHU, Lille, France; 'Université Lille, Inserm, CHU Lille, Service d'ORL, U1192 - PRISM, Lille, France
\end{abstract}

\section{Key words}

Asymmetric hearing loss · Cochlear implants · Quality of life . Contralateral routing of the signal hearing Aids . Bone-anchored hearing system

\begin{abstract}
Introduction: Cochlear implantation is a recent approach proposed to treat single-sided deafness (SSD) and asymmetric hearing loss ( $A H L)$. Several cohort studies showed its effectiveness on tinnitus and variable results on binaural hearing. The main objective of this study is to assess the outcomes of cochlear implantation and other treatment options in SSD/AHL on quality of life. Methods: This prospective multicenter study was conducted in 7 tertiary university hospitals and included an observational cohort study of SSD/AHL adult patients treated using contralateral routing of the sig-
\end{abstract}

karger@karger.com www.karger.com/aud

Karger $\frac{1}{\%}$

GOPEN ACCESS
(C) 2021 The Author(s)

Published by S. Karger AG, Basel

This is an Open Access article licensed under the Creative Commons Attribution-NonCommercial-4.0 International License (CC BY-NC) (http://www.karger.com/Services/OpenAccessLicense), applicable to the online version of the article only. Usage and distribution for commercial purposes requires written permission. nal (CROS) hearing aids or bone-anchored hearing systems (BAHSs) or who declined all treatments, and a randomized controlled trial in subjects treated by cochlear implantation, after failure of CROS and BAHS trials. In total, 155 subjects with SSD or AHL, with or without associated tinnitus, were enrolled. After 2 consecutive trials with CROS hearing aids and BAHSs on headband, all subjects chose any of the 4 treatment options (abstention, CROS, BAHS, or cochlear implant [CI]). The subjects who opted for a $\mathrm{Cl}$ were randomized between 2 arms (Cl vs. initial observation). Six months after the treatment choice, quality of life was assessed using both generic (EuroQoL-5D, EQ-5D) and auditory-specific qualityof-life indices (Nijmegen Cochlear implant Questionnaire [NCIQ] and Visual Analogue Scale [VAS] for tinnitus severity). Performances for speech-in-noise recognition and localization were measured as secondary outcomes. Results: CROS was chosen by 75 subjects, while 51 opted for cochlear im- 
plantation, 18 for BAHSs, and 11 for abstention. Six months after treatment, both EQ-5D VAS and auditory-specific quality-of-life indices were significantly better in the " $\mathrm{Cl}$ " arm versus "observation" arm. The mean effect of the $\mathrm{Cl}$ was particularly significant in subjects with associated severe tinnitus (mean improvement of 20.7 points \pm 19.7 on EQ-5D VAS, 20.4 \pm 12.4 on $\mathrm{NCIQ}$, and $51.4 \pm 35.4$ on tinnitus). No significant effect of the $\mathrm{Cl}$ was found on binaural hearing results. Before/ after comparisons showed that the CROS and BAHS also improved significantly NCIQ scores (for CROS: $+7.7,95 \%$ confidence interval $[95 \% \mathrm{Cl}]=[4.5 ; 10.8]$; for the BAHS: $+14.3,95 \%$ $\mathrm{Cl}=[7.9 ; 20.7])$. Conclusion: Cochlear implantation leads to significant improvements in quality of life in SSD and AHL patients, particularly in subjects with associated severe tinnitus, who are thereby the best candidates to an extension of $\mathrm{Cl}$ indications.

(c) 2021 The Author(s).

Published by S. Karger AG, Basel

\section{Introduction}

Single-sided deafness (SSD) affects approximately $1 \%$ of the adult population, with an estimated incidence of 200 new cases per million per year [Baguley et al., 2006]. In a strict definition [Vincent et al., 2015; Van de Heyning et al., 2016], it refers to the condition where hearing is normal or near-normal in 1 ear, while the other ear presents severe to profound deafness. In asymmetric hearing loss (AHL), unaided hearing thresholds in the better ear are between 30 and $60 \mathrm{~dB}$ HL (decibel hearing level) and may be compensated by a conventional hearing aid. Both conditions lead to binaural deficits, with difficulties for localizing sound sources and for understanding speech in noise, which deteriorate global quality of life. The presence of an associated disabling tinnitus may further impair the patients' daily life and become their first complaint [Van de Heyning et al., 2008].

Treatment of SSD/AHL may rely on the transfer of the sound coming to the poor ear to the better ear using whether Bluetooth technology in contralateral routing of the signal (CROS) hearing aids, whether bone conduction properties of the skull with a bone-anchored hearing system (BAHS). These options may be tried before being adopted, but a significant proportion of SSD/AHL subjects remain untreated after trial [Desmet et al., 2012; Saroul et al., 2014; Pennings et al., 2011].

Cochlear implantation is another approach where the poor ear is stimulated and binaural hearing might thereby be restored. Further, the electrical stimulation by the cochlear implant (CI) has been shown to reduce the tin- nitus when associated with profound deafness [(Baguley and Atlas, 2007); Quaranta et al., 2004]. First meta-analyses and systematic reviews on that topic emphasized the effectiveness of the CI on tinnitus but pointed out the need for controlled trials to provide more robust evidence of CI effects on binaural hearing and quality of life [Blasco and Redleaf, 2014; Vlastarakos et al., 2014; van Zon et al., 2015]. They also acknowledged the difficulty of conducting such studies because of the important intrinsic differences between the existing treatments.

In the present study, each treatment option (abstention, CROS, BAHS, and CI) could be chosen after successive trials of CROS hearing aids and BAHSs on a headband. This prospective national multicenter study included a randomized controlled trial (RCT) in the group of subjects who opted for cochlear implantation, aiming at assessing the outcomes of the CI in SSD/AHL on hearing performances but also on quality of life, after failure of more conservative treatments. In addition, it describes and compares results obtained with the other options used to handle this condition, that is, CROS hearing aids, BAHSs, or abstention.

\section{Methods}

This national multicenter study was conducted in 7 tertiary referral centers in France, between 2014 and 2018, and was divided into 2 parts (see [Marx et al., 2019] for details). The first part is a prospective, descriptive observational cohort study, with a 6-month follow-up for SSD/AHL adult subjects who chose to be treated by CROS hearing aids, or by BAHSs, or by CI and patients who declined all the options, after 2 consecutive trials of the CROS and BAHS. The second part is an open-label randomized controlled clinical trial for subjects with SSD/AHL who chose to be treated by the CI after failure of both CROS and BAHS trials, in 2 parallel arms: observation for 6 months versus cochlear implantation. The subjects randomized in the "observation" arm could benefit from a cochlear implantation procedure once this 6-month observation initial period had ended. This research has been registered in ClinicalTrials.gov (http://www.clinicaltrials.gov/), 29 July 2014, under no. NCT02204618.

\section{Participants}

Adults older than 18 years, affiliated to social security, were included with SSD or AHL documented using pure-tone average (PTA) on pure-tone audiometry. Because all subjects had to be theoretical candidates to a CI, patients with SSD/AHL due to a vestibular schwannoma or to major modifications of cochlear anatomy were excluded. There was no eligibility criterion applied on the duration of deafness, or an associated tinnitus, to account for the heterogeneity of this population. PTA in the better ear was between 0 and $60 \mathrm{~dB}$ HL.
Cochlear Implantation and Other

Treatments in Asymmetric Hearing Loss
Audiol Neurotol 2021;26:414-424

DOI: $10.1159 / 000514085$ 


\section{Interventions}

We defined 4 groups depending on the treatment option chosen after the consecutive trials of CROS hearing aids and BAHSs: abstention, CROS, BAHS, and randomized for CI (RCI). The subjects successively tried CROS hearing aids and BAHSs before choosing which treatment they opted for. This choice was mainly based on the subjective outcomes reported after the 2 trials but also guided by the auditory outcomes obtained with each device, the recommendations of the corresponding manufacturer, and the counselling of the physician.

In the group CROS, a Phonak CROS system was fitted by an experienced audiologist, using corresponding fitting software (Phonak ${ }^{\circledR}$ Target 3.0). The subjects with AHL were fitted with BiCROS systems to correct hearing loss in the better ear. In the group BAHS, Baha $110^{\circledR}$ (Cochlear Ltd) and Ponto ${ }^{\circledR}$ (Oticon medical) were adapted on the abutment 3-5 weeks after the surgical procedure and fitted by an audiologist according to the company's guidelines. Both Cochlear Ltd and Oticon medical recommend the use of BAHSs in SSD, but not AHL. In the group RCI, patients were randomized to a 6-month "observation" arm or an immediate cochlear implantation "CI" arm. The CI, supporting 12-22 intracochlear electrodes, was placed after the traditional surgical procedure, including mastoidectomy, posterior tympanotomy, and round window insertion or cochleostomy. Four major companies were represented (Advanced Bionics ${ }^{\circledR}$, Cochlear $^{\circledR}, \operatorname{MedEl}^{\circledR}$, and Oticon Medical ${ }^{\circledR}$ ).

\section{Primary and Secondary Outcomes}

First, the present article reports the results of CI in SSD/AHL compared to observation in terms of quality of life after a 6-month follow-up, both from a generic (EuroQoL-5D, EQ-5D) and an auditory-specific perspective (Nijmegen Cochlear Implant Questionnaire, NCIQ; Visual Analogue Scale [VAS] for tinnitus severity), as in previous national multicenter studies [Molinier et al., 2009]. The EQ-5D [EuroQol Group, 1990] is recommended by a national French agency (Haute Autorité de Santé) for generic evaluation of quality of life [Haute Autorité de la Santé (HAS), 2014; Haute Autorité de Santé (HAS), 2016]. It is a self-administered, generic, and multidimensional questionnaire including a descriptive component and a VAS (EQ-VAS). The descriptive component is composed of 5 dimensions (mobility, autonomy, daily life activities, pain, and anxiety/depression) described by 3 levels. The NCIQ [Hinderink et al., 2000] contains 60 questions exploring 6 areas concerned with the quality of auditory perception (basic perception, complex perception, speech production, self-esteem, social activities, and interactions). The VAS used to assess tinnitus severity is a $17-\mathrm{cm}$ plastic ruler with a vertical arrow on one side and a graduated scale on the other side $(0-10 \mathrm{~cm})$, and discomfort related to tinnitus is thus self-determined by the patient, quoted between 0 and 100 points.

Second, hearing outcomes were assessed after a 6-month follow-up using a test for speech recognition in noise (FraMatrix) and a horizontal localization test. The FraMatrix test (Jansen et al., 2012] relies on an adaptive procedure using 3 loudspeakers surrounding the subject $\left(-60^{\circ}, 0^{\circ}\right.$, and $\left.+60^{\circ}\right)$. The speech signal was presented at a fixed level of $65 \mathrm{~dB}$ SPL, and the level of the competing noise was progressively adapted to obtain the signal-to-noise ratio in decibels for $50 \%$ correct word recognition (SNR50). The subjects were tested in 3 different spatial conditions: with speech presented to the poor ear and noise to the better ear (SpeNbe), with speech and noise collocated and presented from the loudspeaker in front of the subject, and with noise presented to the poor ear and speech to the better ear (SbeNpe). Horizontal localization was assessed using an array of 7 loudspeakers located at $30^{\circ}$ intervals from $-90^{\circ}$ to $+90^{\circ}$, in a frontal semicircle diameter of $1.2 \mathrm{~m}$ at the subject's head level [Slattery, and Middlebrooks, 1994; Vannson et al., 2017]. The stimuli consisted in two 150-ms white noises, separated by a 50 -ms interval, and presented 63 times. After each presentation, a 2-s period allowed the subject to indicate orally the loudspeaker identified as the sound source. Root mean square error (RMSE) was the main measurement for localization performance.

\section{Sample Size Calculation}

In order to detect an improvement of 0.8 standard deviation on quality of life, with a two-sided 5\% significance level and a power of $80 \%, 25$ patients per arm were necessary in the group RCI. The total number of subjects, including the observational part of the study, was estimated based on the expected rate of trial failures reported in the literature (up to $45 \%$ for BAHSs in [Desmet et al., 2012]). Altogether, at least 150 subjects needed to be included.

\section{Randomization Procedure}

Randomization of CI versus initial observation was based on a 1:1 ratio and was stratified per center. The allocation sequence was randomly and automatically generated (Stata SE 11.2, ralloc procedure). There was no blinding procedure in this open-label trial, but the allocation sequence was unknown to all investigators.

\section{Statistical Analysis}

We applied an intention-to-treat analysis on the nonmissing values at the 6-month follow-up. Quantitative variables were described as mean \pm standard deviation. We compared primary and secondary outcomes at 6 months of the follow-up between the 2 randomized arms using Wilcoxon rank-sum tests. Then, we applied linear regressions adjusted for baseline values of the outcomes and robust estimators of variance to compare the 2 randomized arms and test interactions terms between the intervention and deafness duration or PTA in the better ear. Finally, linear regressions adjusted for baseline values (and robust estimators of variance) were used to estimate the mean evolutions from baseline to the 6-month follow-up and to compare these evolutions among the 5 groups (abstention, CROS, BAHS, “observation" in the RCI group, and CI in the RCI group).

\section{Results}

\section{Patients Characteristics}

Overall, 155 subjects were included in this study. PTA was $105.4( \pm 22.9) \mathrm{dB}$ HL in the poor ear and $27.1( \pm 19)$ $\mathrm{dB}$ HL in the better ear, with $104 \mathrm{SSD}(67.1 \%)$ and $51 \mathrm{AHL}$ subjects (32.9\%). After the 2 trials for CROS and BAHSs, 75 subjects opted for CROS hearing aids (group CROS), 18 for BAHSs (group BAHS), and 51 were randomized for a CI (group RCI). In the latter, 25 participants were randomized in the "CI" arm and 26 in the "observation" 
Table 1. Demographic characteristics per group

\begin{tabular}{|c|c|c|c|c|}
\hline & $\begin{array}{l}\text { Abstention } \\
(n=11)\end{array}$ & $\begin{array}{l}\text { CROS } \\
(n=75)\end{array}$ & $\begin{array}{l}\text { BAHS } \\
(n=18)\end{array}$ & $\begin{array}{l}\text { CI } \\
(n=51)\end{array}$ \\
\hline Sex & $8 \mathrm{M} / 3 \mathrm{~F}$ & $35 \mathrm{M} / 40 \mathrm{~F}$ & $7 \mathrm{M} / 11 \mathrm{~F}$ & $21 \mathrm{M} / 30 \mathrm{~F}$ \\
\hline Age, mean $\pm S D$, years & $53.1 \pm 20.2$ & $51.9 \pm 13.8$ & $49.7 \pm 14.5$ & $55.1 \pm 11.4$ \\
\hline Deafness side & $4 \mathrm{R} / 7 \mathrm{~L}$ & $39 \mathrm{R} / 36 \mathrm{~L}$ & $9 \mathrm{R} / 9 \mathrm{~L}$ & $30 \mathrm{R} / 21 \mathrm{~L}$ \\
\hline \multicolumn{5}{|l|}{ Etiology } \\
\hline Idiopathic SSNHL & 1 & 24 & 7 & 23 \\
\hline Meniere's disease & 1 & 8 & & 2 \\
\hline Labyrinthine trauma & & 9 & 1 & 3 \\
\hline Labyrinthitis & & 4 & 4 & 3 \\
\hline Other & 9 & 30 & 6 & 20 \\
\hline \multicolumn{5}{|l|}{ Deafness duration } \\
\hline$<3$ years & 2 & 33 & 9 & 25 \\
\hline Between 3 and 5 years & 1 & 6 & 2 & 2 \\
\hline Between 5 and 10 years & 1 & 8 & 1 & 6 \\
\hline Between 10 and 30 years & 6 & 15 & 2 & 13 \\
\hline$>30$ years & 1 & 12 & 4 & 5 \\
\hline Missing data & & 1 & & \\
\hline PTA better ear dB HL (mean \pm SD) & $27.5 \pm 22.2$ & $26.8 \pm 20.2$ & $21.8 \pm 16.7$ & $29.2 \pm 17.5$ \\
\hline PTA poor ear dB HL (mean \pm SD) & $106.8 \pm 19$ & $106.9 \pm 26.1$ & $110.9 \pm 17.2$ & $101.3 \pm 20.6$ \\
\hline
\end{tabular}

M, male; F, female; SD, standard deviation; R, right; L, left; SSNHL, sudden sensorineural hearing loss; PTA, pure-tone average; dB HL, decibel hearing level; BAHS, bone-anchored hearing system; CI, cochlear implant; 95\% CI, 95\% confidence interval; CROS, contralateral routing of the signal.

arm. Eleven subjects declined all options (group abstention). Baseline characteristics are described in Table 1, and the flowchart study is illustrated on Figure 1.

\section{Loss to Follow-Up and Missing Data}

One participant was excluded before inclusion because a vestibular schwannoma was found in the poor ear. At the 6-month follow-up visit, in the group CROS, 2 subjects were lost to follow-up and 5 withdrew their consent for personal reasons. In the group BAHS, 1 subject was explanted, 1 was lost to follow-up, and 1 was excluded by the local investigator. In the group RCI, that is, the "CI" arm, 1 subject was lost to follow-up and 3 were excluded due to medical reasons. One subject was diagnosed with dementia, and 2 subjects presented postoperative local infections requiring extra visits and treatments. One of these 2 subjects was finally explanted and reimplanted, and the infection resolved in the other one. In the group $\mathrm{RCI}$, that is, the "observation" arm, 3 subjects were excluded from the analyses: 1 for a major protocol violation, 1 withdrew his consent, and 1 was lately diagnosed with a small vestibular schwannoma. Two subjects withdrew their consent, and 2 were lost to follow-up in group abstention.

Cochlear Implantation and Other

Treatments in Asymmetric Hearing Loss

\section{CI versus Observation in SSD/AHL at 6-Month}

Follow-Up

Quality-of-Life Measurements

There was no significant difference between the 2 arms (CI vs. observation) for the scores of EQ-5D on the descriptive component (77.6 \pm 27.3 in the "CI" arm vs. $77.7 \pm 18.1$ in the "observation" arm). In contrast and as shown in Figure 2, EQ-VAS evaluation was significantly higher in the "CI" $(81.6 \pm 19.4)$ than the "observation" arm (71.8 \pm 16.7$)$. Likewise, the NCIQ score was significantly higher in "CI" $(68.7 \pm 16)$ than the "observation" arm $(55.1 \pm 15.8)$, with a trend for an interaction between NCIQ improvement and poor PTA in the better ear ( $p=$ $0.06)$.

The VAS for tinnitus severity also showed better results in the "CI" arm, with a mean reduction of 30.5 $( \pm 36.5)$ points, whereas the mean score remained stable in the "observation" arm $(-0.8 \pm 24.9)$. These results persisted after baseline adjustment, with a mean effect of the CI versus initial observation of +11.27 points on EQ-VAS $(p=0.01)$, of +12.4 points on the NCIQ $(p=0.006)$ and a reduction of 20.02 points on tinnitus severity $(p=0.02)$. Effects were particularly significant for subjects with incapacitating tinnitus (VAS $\geq 60)$. These cases $(n=20)$ ac- 


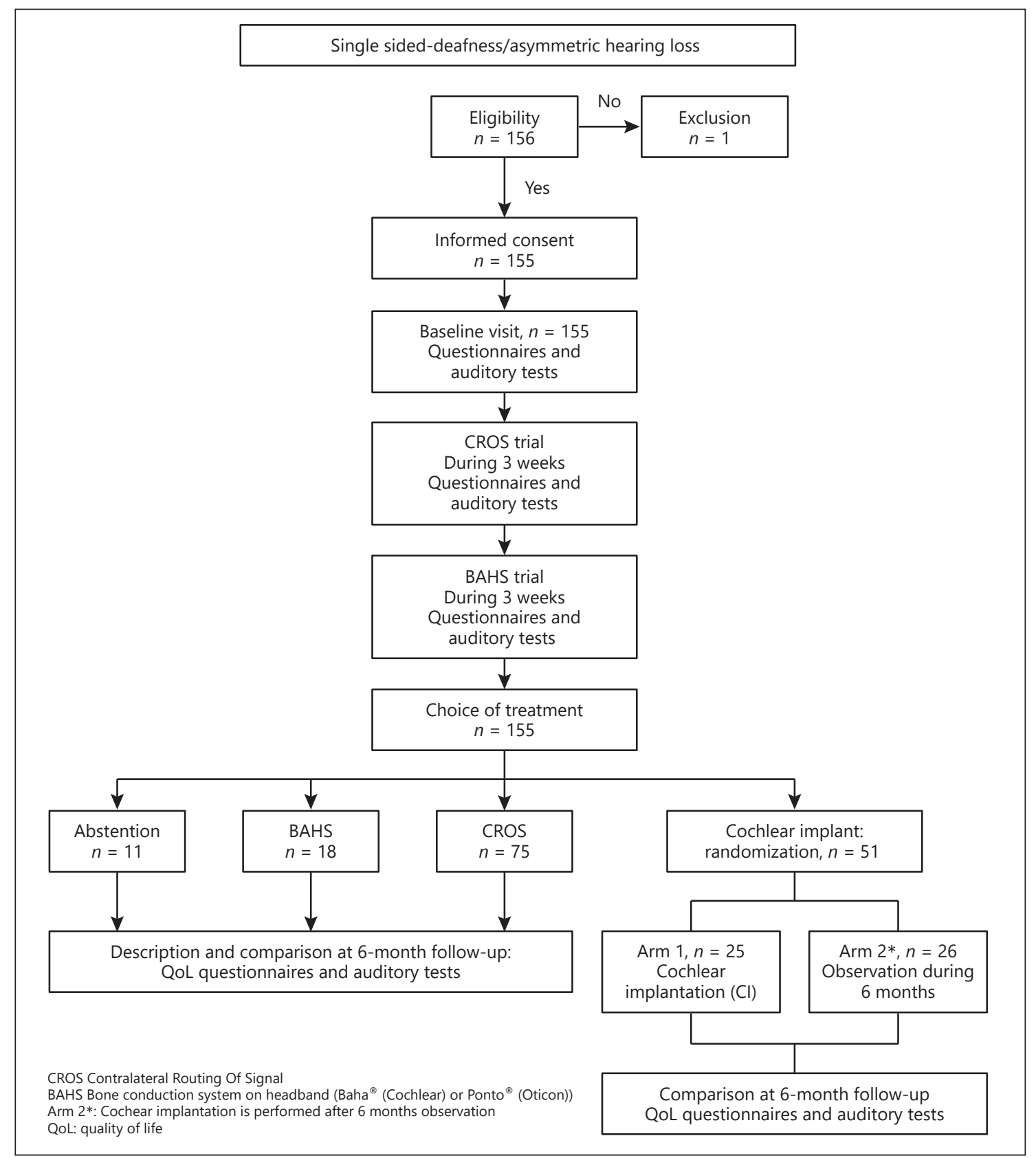

Fig. 1. Flowchart of the study. Number of subjects included, allocated to intervention, lost during follow-up with explanation for the dropouts, and number included in the statistical analysis. SSD, single-sided deafness; AHL, asymmetric hearing loss; CROS, contralateral routing of the signal; BAHS, bone-anchored hearing system; CI, cochlear implant.

counted for the major part of EQ-VAS improvement $(+20.7$ points \pm 19.7 in the "CI" arm vs. +3.9 points \pm 14.1 in the "observation" arm) and of tinnitus relief with a mean reduction of $51( \pm 35.4)$ points in the "CI" arm versus $11.8( \pm 22.4)$ points in the "observation" arm (see Fig. 2d).

\section{Hearing Outcomes}

As illustrated in Table 2, there was no difference between the 2 randomized arms for speech recognition in noise or horizontal localization at 6 months, even after baseline adjustment. In fact, the slight SNR50 difference between the 2 arms observed in the SpeNbe condi- 


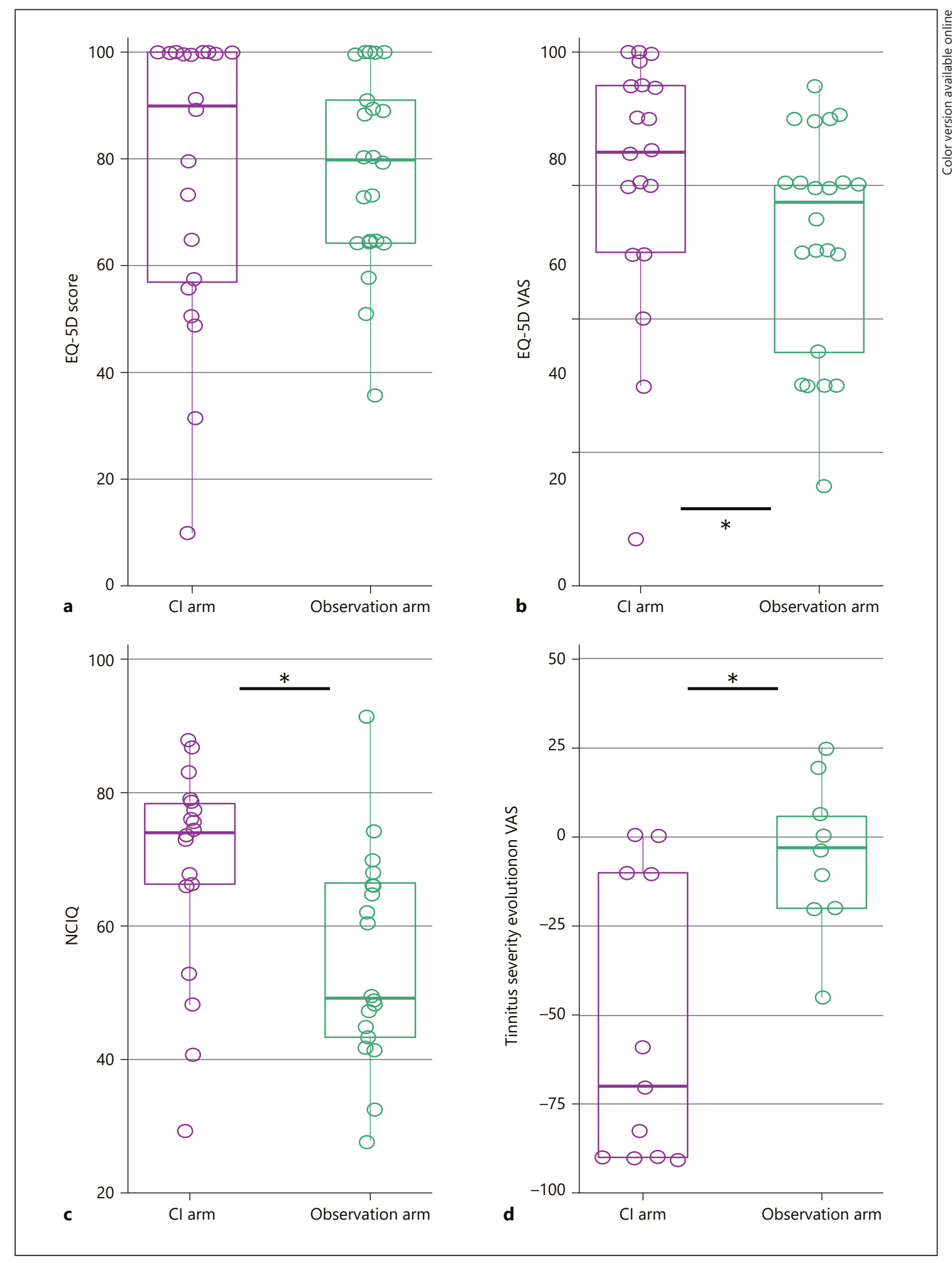

Fig. 2. a-c Comparisons between the "CI" arm and "observation" arm in all subjects of the group RCI, for EQ5D score (a), EQ-5D VAS (b), NCIQ score (c), at 6-month follow-up. d Comparison between the 2 arms in subjects with severe tinnitus (i.e., baseline VAS $\geq 60, n=20)$. * Statistical significant differences $(p<0.05)$. CI, cochlear implant; EQ-5D, EuroQoL-5D; NCIQ, Nijmegen Cochlear implant Questionnaire; VAS, Visual Analogue Scale; RCI, randomized for CI. 
Table 2. Hearing outcomes at 6-month follow-up in the group RCI

\begin{tabular}{|c|c|c|c|c|c|c|}
\hline & $\begin{array}{l}\text { Arm CI } \\
(N=21) \\
(\text { mean } \pm \text { SD })\end{array}$ & $\begin{array}{l}\text { Arm } \\
\text { observation } \\
(N=23) \\
(\text { mean } \pm \mathrm{SD})\end{array}$ & $p$ value* & \multicolumn{3}{|c|}{$\begin{array}{l}\text { CI versus "observation" mean difference } \\
\text { adjusted for baseline value, estimations at } \\
\text { 6-month follow-up }{ }^{\dagger}\end{array}$} \\
\hline \multicolumn{7}{|c|}{ Speech-in-noise recognition (SNR50 in dB at baseline) } \\
\hline SpeNbe & $3.7 \pm 9.7$ & $5.6 \pm 9.6$ & & & & \\
\hline \multicolumn{7}{|c|}{ Speech-in-noise recognition (SNR50 in $\mathrm{dB}$ at 6-month follow-up) } \\
\hline SpeNbe & $-1.0 \pm 8$ & $2.2 \pm 7.9$ & 0.05 & -2.35 & $(-6.59 ; 1.89)$ & 0.27 \\
\hline SONO & $-0.9 \pm 4.3$ & $0 \pm 3.2$ & 0.19 & -1.17 & $(-3.14 ; 0.80)$ & 0.24 \\
\hline SbeNpe & $-3.8 \pm 4.5$ & $-3.1 \pm 6$ & 0.64 & -0.31 & $(-3.22 ; 2.61)$ & 0.83 \\
\hline Localization ( $\mathrm{RMSE}^{\circ}{ }^{\circ}$ at baseline) & $73.2 \pm 20.7$ & $76.6 \pm 20$ & & & & \\
\hline Localization (RMSE in ${ }^{\circ}$ at 6 -month follow-up) & $63.3 \pm 23.4$ & $63.8 \pm 19.3$ & 0.80 & 0.22 & $(-14.63 ; 15.07)$ & 0.98 \\
\hline
\end{tabular}

tion $(-1 \pm 8$ in the "CI" arm vs. $2.2 \pm 7.9$ in the "observation" arm, $p=0.05$ ) did not persist after baseline adjustments. In the "CI" arm, no significant interaction was found between the SNR50 value evolution, whatever the condition, and deafness duration, or PTA in the better ear $(p>0.2)$.

\section{Results with Different Options (Abstention, CROS, $B A H S$, and CI)}

Mean intraindividual evolutions between baseline and 6-month follow-up visit were estimated within each treatment group (see Table 3). Regarding EQ-5D, the largest change from baseline was observed for the VAS scale in the "CI" arm of the group RCI (mean increase of +11.72 , $95 \%$ confidence interval $[95 \% \mathrm{CI}]=[4.70 ; 18.73])$. NCIQ scores were mostly improved in groups CROS $(+7.7,95 \%$ $\mathrm{CI}=[4.5 ; 10.8])$, BAHS $(+14.3,95 \% \mathrm{CI}=[7.9 ; 20.7])$, and in the "CI" arm in the group RCI $(+15.2,95 \% \mathrm{CI}=[8.8$; 21.6]). NCIQ score evolution was smaller in the group abstention $(+1.9,95 \% \mathrm{CI}=[-8.0 ; 11.8])$ and the "observation" arm of the group RCI $(+2.6,95 \% \mathrm{CI}=[-2.9 ; 8.1])$. Tinnitus severity was clearly reduced in the "CI" arm of the group RCI $(-25.8,95 \% \mathrm{CI}=[-36.2 ;-15.4])$. Tinnitus severity was also decreased in the group BAHS by -23.4 , $95 \% \mathrm{CI}=[-35.8 ;-11.1]$. Mean SNR50 improved between baseline measurements and 6-month follow-up visit in CROS and the 2 arms in the group RCI, whatever the con- dition (except from SbeNpe in the group CROS). Localization performance measured by root mean square error was reduced in the group CROS $\left(+7.72^{\circ}, 95 \% \mathrm{CI}=\left[2.71^{\circ}\right.\right.$; $\left.12.73^{\circ}\right]$ ) but tended to be improved in the group BAHS and the 2 randomized groups.

The primary and secondary outcomes at 6-month follow-up in each group (abstention, CROS, BAHS, and "observation" arm of the RCI group) were then compared to the outcomes in the "CI" arm of the RCI group (serving as the reference), after baseline adjustment (see Table 3). The subjects in the group CROS reported poorer EQ-VAS scores $(-12.7$ points; $95 \% \mathrm{CI}=[-20.9 ;-4.5]$, $p=0.003)$. Groups CROS and abstention showed poorer scores for NCIQ (for CROS: -7.5 points; $95 \% \mathrm{CI}=[-14.6$; $-0.4], p=0.04$; for abstention: -13.3 points, $95 \% \mathrm{CI}=$ $[-25.1 ;-1.5], p=0.03)$. The group CROS showed poorer results for the FraMatrix test in the SbeNpe condition with higher SNR50 values $(+2.62 \mathrm{~dB}$; 95\% CI $=[0.16$; 5.09]; $p=0.04)$ and for horizontal localization $\left(+16.56^{\circ}\right.$; $\left.95 \% \mathrm{CI}=\left[6.12^{\circ} ; 27.00^{\circ}\right] ; p=0.002\right)$.

\section{Discussion}

To our knowledge, our study reports one of the first RCTs demonstrating efficacy of the CI in SSD/AHL on generic and auditory-specific quality of life. Results on 
Table 3. Comparisons of the different treatment groups with the arm CI in group RCI as reference

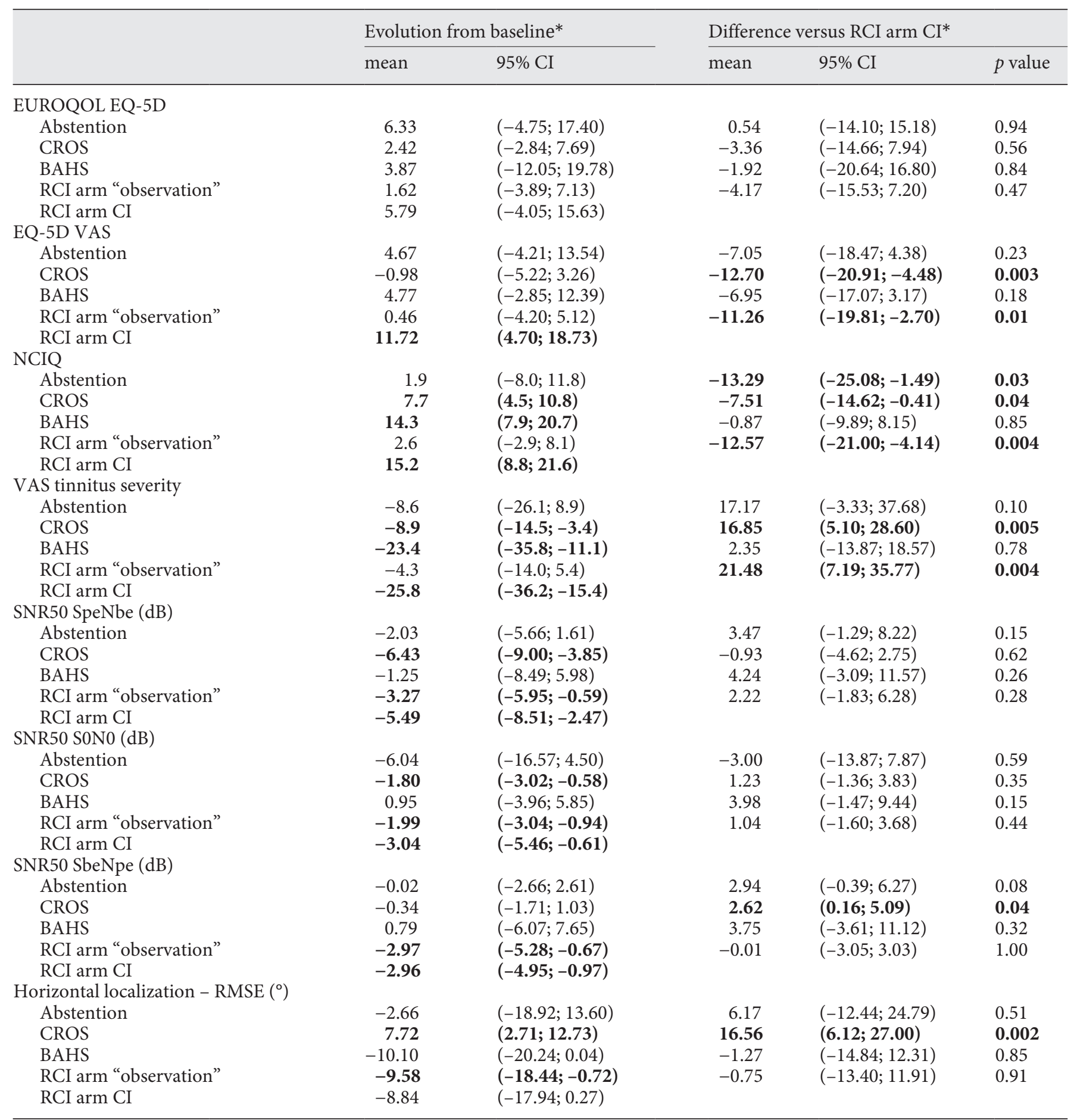

SpeNbe, speech presented to the poor ear and noise to the better ear; SbeNpe, noise presented to the poor ear and speech to the better ear; 95 CI, 95\% confidence interval; BAHS, bone-anchored hearing system; CI, cochlear implant; EQ-5D, EuroQoL-5D; NCIQ, Nijmegen Cochlear implant Questionnaire; VAS, Visual Analogue Scale; CROS, contralateral routing of the signal; RCI, randomized for CI; SNR50, signal-to-noise ratio for 50\% correct word recognition; S0N0, speech and noise collocated and presented from the loudspeaker in front of the subject; RMSE, root mean square error. ${ }^{*}$ Mean differences, $95 \%$ CIs, and $p$ values are estimated using linear regressions adjusted for the baseline value of the outcome, with a robust estimator of variance. Significant differences are indicated in bold.

Cochlear Implantation and Other

Treatments in Asymmetric Hearing Loss
Audiol Neurotol 2021;26:414-424 DOI: $10.1159 / 000514085$ 
tinnitus severity were expected based on pioneering work of Van de Heyning [Van de Heyning et al., 2008], followed by several teams [Buechner et al., 2010; Arts et al., 2015; Arts et al., 2016], who showed an important and consistent relief from tinnitus in severely impaired SSD subjects. Whether peripheral or central, the processes underlying this effect remain unclear, but its consistency approximates $60-80 \%$ of SSD/AHL cases treated by the CI. It was also the case in our study, and this explains why recent meta-analyses identified it as the most plausible benefit of the $\mathrm{CI}$ in this indication.

The assessment of quality of life is recommended in consensus articles on SSD/AHL and should associate generic and disease-specific questionnaires [Vincent et al., 2015; Van de Heyning et al., 2016]. Health Utility IndexIII and Speech, Spatial and hearing qualities Questionnaire are the most often recommended scales in this line [Kitterick et al., 2015; Kitterick et al., 2016; Ramakers et al., 2016]. The first one is more likely to reflect changes in quality of life after a treatment for hearing or vision than other generic questionnaires. The second one provides a subjective evaluation of binaural hearing abilities, whereas the NCIQ rather explores the global hearing quality. Here, the decision to opt here for EQ-5D and NCIQ was mainly driven by guidelines from national authority [Haute Autorité de la Santé (HAS), 2014; Haute Autorité de Santé (HAS), 2016). Although EQ-5D is less sensitive than Health Utility Index-III to hearing changes, in our study, its VAS component was significantly improved in the "CI" arm compared to the "observation" arm, especially in subjects with tinnitus severity $\geq 60 / 100$. Altogether, our results emphasize the remarkable effects of the CI on quality of life of SSD/AHL subjects with associated incapacitating tinnitus.

Effects of CI on binaural abilities of SSD/AHL subjects remain controversial. In our study, there was no significant evolution of binaural performance after cochlear implantation. Intragroup comparisons showed an improvement of SNR50 in SpeNbe and SbeNpe conditions between the inclusion and the 6-month follow-up in the "CI" arm. But such improvement was also demonstrated in the "observation" arm (see Table 3), which emphasizes the existence of learning effects after repeated exposition to the speech material used for speech in noise recognition tests. The absence of a significant auditory effect in the "CI" arm, for localization accuracy and speech recognition in noise, might be related to the short follow-up of 6 months after cochlear implantation, and/or to the deafness duration, which exceeded 10 years in more than onethird of the subjects in the RCI group. Indeed, several valuable reports concluded that both localization accuracy and speech in noise recognition improved with CI experience [Arndt et al., 2011; Távora-Vieira et al., 2015]. In fact, recent studies focused on SSD/AHL subjects with a deafness duration usually $<10$ years and suggested a progressive improvement, though variable, of both skills during the first 3 years following CI [Mertens et al., 2015; Sullivan et al., 2019], which may be delayed even after 5 years [Sullivan et al., 2019]. Restoration of interaural level differences after CI might play a key role when localization is shown to improve [Távora-Vieira et al., 2015; Dirks et al., 2019]. The influence of CI on binaural effects is still under debate. Most reports demonstrate an increase in the head shadow effect when speech is presented to the poor ear, also possibly related to the improved perception of interaural-level differences [Vermeire and Van de Heyning, 2009; Arndt et al., 2011; Mertens et al., 2015], but the evolution of the summation effect and binaural unmasking seems more uncertain. Sullivan et al. (2019) did not find any modification of these 2 latter effects, even after long CI experience, whereas Mertens' study reported significant improvement [Mertens et al., 2015], especially in subjects with AHL. The integration of both electric and acoustic hearing modalities might be facilitated in AHL because of the degraded acoustic sound coming to the better ear, usefully completed by the CI. In our study, the number of AHL subjects in the RCI group was too limited to allow robust comparisons between the 2 arms, excluding the tinnitus severity factor.

To date, only one study compared CROS, BAHS, and CI successively used to treat the same SSD subjects [Arndt et al., 2011]. This study demonstrated the plausible superiority of CI for localization and speech in noise when speech is presented to the poor ear. Our observational cohort study does not show such effects on binaural performance but showed consistently poorer results obtained in the group CROS in reference to the arm "CI." Surprisingly, in our study, the group BAHS showed an improvement of tinnitus at the 6-month follow-up. This effect was mainly driven by 3 subjects with significant airbone gap in the poor or the better ear. CROS or BAHS was not included as randomization arms in our RCT because they are not recommended as SSD/AHL standard of care in France and because consensus articles identify them as first-line treatments which should be tried before considering cochlear implantation. However, much robust evidence is expected from an RCT conducted by Peters [Peters et al., 2015] where a "CI" arm is compared to both CROS and BAHS, and from a longitudinal study led by Kitterick [Kitterick et al., 2014]. 


\section{Strengths and Limitations}

This study comprises an RCT examining the effects of CI on quality of life of SSD/AHL subjects. The evidence in cases of associated incapacitating tinnitus is therefore robust. It also comprises a cohort study which provides an overview of this heterogeneous condition and analyzes the same outcomes obtained with the other potential treatments.

However, the sample size included in the RCT remains relatively small and might have limited the differences existing between the 2 arms, especially for speech recognition in noise. In addition, the 6-month follow-up was probably insufficient to show effects of CI on binaural abilities, which require experience to settle. The follow-up of this population is planned to provide information regarding long-term hearing outcomes after CI. Likewise, long-term retention and usage of all treatments chosen in this study should carefully be looked at to provide additional evidence of their impact.

\section{Conclusions}

The observational part of this study suggested that CROS hearing aids, though more often chosen, provided poorer results than CI to treat SSD/AHL. Our RCT demonstrated that CI significantly improved quality of life, particularly in subjects with severe tinnitus associated with profound hearing loss, when CROS and BAHS trials are ineffective. The improvement was shown both on generic and auditory-specific indices. Therefore, this population might be the main target of expanded indications for cochlear implantation.

\section{Acknowledgements}

We sincerely thank Ms. Soumia Taoui for her precious help in coordinating all the centers and the numerous institutions involved. We are grateful to all the participants who took part in this study, and we wish to extend our sincere thanks to the collaborating clinical teams, research associates, and audioprosthesists at the university hospitals of Toulouse, Paris, Lille, Montpellier, Tours, Rennes, and Grenoble.

\section{Statement of Ethics}

All of the authorizations required in accordance with French legislation were obtained for this study, "Agence Nationale de Sécurité du Médicament" (ANSM, National Agency of Drug Safety) (authorization dated May 2014), and approved by the "Commission Nationale Informatique et Libertés" (CNIL, French National Agency regulating Data Protection). This study was presented to the southwest and overseas territories' Ethics Committee on May 2014 under the reference CPP14-020/2014-A00533-44. All the patients taking part in this clinical trial have received verbal and written information and have been able to ask questions about the study. They willingly signed an informed consent form before starting the trial.

\section{Conflict of Interest Statement}

The authors have no conflicts of interest to declare.

\section{Funding Sources}

This study is financed by the French Ministry of Health (tender for research focusing on medical costs PRME2013 number 137053 $10)$.

\section{Authors Contributions}

M.M., B.L., O.D., and B.F. designed the study. M.M. is the lead author responsible for manuscript drafting. B.L. is the author responsible for statistical analyses. All other authors included participants to the study, and all authors provided significant inputs to the first version of the manuscript. All authors read and approved the final version of the manuscript.

\section{References}

Arndt S, Aschendorff A, Laszig R, Beck R, Schild C, Kroeger S, et al. Comparison of pseudobinaural hearing to real binaural hearing rehabilitation after cochlear implantation in patients with unilateral deafness and tinnitus. Otol Neurotol. 2011;32(1):39-47.

Arts RAGJ, George ELJ, Griessner A, Zierhofer C, Stokroos RJ. Tinnitus suppression by intracochlear electrical stimulation in single-sided deafness: a prospective clinical trial: part I. Audiol Neurootol. 2015;20:294-313.
Arts RAGJ, George ELJ, Janssen M, Griessner A, Zierhofer C, Stokroos RJ. Tinnitus suppression by intracochlear electrical stimulation in single sided deafness: a prospective clinical trial: follow-up. PLoS One. 2016;11:e0153131.

Baguley DM, Atlas MD. Cochlear implants and tinnitus. Prog Brain Res. 2007;166:347-55.

Baguley DM, Bird J, Humphriss RL, Prevost AT. The evidence base for the application of contralateral bone anchored hearing aids in acquired unilateral sensorineural hearing loss in adults. Clin Otolaryngol. 2006;31(1):6-14.
Blasco MA, Redleaf MI. Cochlear implantation in unilateral sudden deafness improves tinnitus and speech comprehension: meta-analysis and systematic review. Otol Neurotol. 2014; 35(8):1426-32.

Buechner A, Brendel M, Lesinski-Schiedat A, Wenzel G, Frohne-Buechner C, Jaeger B, et al. Cochlear implantation in unilateral deaf subjects associated with ipsilateral tinnitus. Otol Neurotol. 2010;31(9):1381-5.
Cochlear Implantation and Other

Treatments in Asymmetric Hearing Loss
Audiol Neurotol 2021;26:414-424

DOI: $10.1159 / 000514085$ 
Desmet J, Bouzegta R, Hofkens A, De Backer A, Lambrechts P, Wouters K, et al. Clinical need for a Baha trial in patients with single-sided sensorineural deafness. Analysis of a Baha database of 196 patients. Eur Arch Otorhinolaryngol. 2012;269(3):799-805.

Dirks C, Nelson PB, Sladen DP, Oxenham AJ. Mechanisms of localization and speech perception with colocated and spatially separated noise and speech maskers under single-sided deafness with a cochlear implant. Ear Hear. 2019 Nov/Dec;40(6):1293-306.

EuroQol Group. EuroQol: a new facility for the measurement of health-related quality of life. Health Policy. 1990;16(3):199-208.

Haute Autorité de la Santé. Valeurs de référence pour l'évaluation économique en santé [Internet] 2014 [cited 2018 Jul 12]. Available from: https://www.has-sante.fr/portail/upload/docs/application/pdf/2014--12/valeurs_ de_reference_vf.pdf.

Haute Autorité de Santé. Choix méthodologiques pour l'analyse de l'impact budgétaire à la HAS [Internet] 2016 [cited 2018 Jul 12]. Available from: https://www.has-sante.fr/portail/upload/docs/application/pdf/2016--12/guide_ methodologique_choix_methodologiques_ pour_lanalyse_de_limpact_budgetaire_a_la_ has_.pdf.

Hinderink JB, Krabbe PF, Van Den Broek P. Development and application of a health-related quality-of-life instrument for adults with cochlear implants: the Nijmegen cochlear implant questionnaire. Otolaryngol Head Neck Surg. 2000;123:756-65.

Jansen S, Luts H, Wagener KC, Kollmeier B, Del Rio M, Dauman R, et al. Comparison of three types of French speech-in-noise tests: a multicenter study. Int J Audiol. 2012;51(3):164-73.

Kitterick PT, Lucas L, Smith SN. Improving health-related quality of life in single-sided deafness: a systematic review and meta-analysis. Audiol Neurootol. 2015;20(Suppl 1):7986.

Kitterick PT, O'Donoghue GM, EdmondsonJones M, Marshall A, Jeffs E, Craddock L, et al. Comparison of the benefits of cochlear im- plantation versus contra-lateral routing of signal hearing aids in adult patients with single-sided deafness: study protocol for a prospective within-subject longitudinal trial. BMC Ear Nose Throat Disord. 2014;14:7.

Kitterick PT, Smith SN, Lucas L. Hearing instruments for unilateral severe-to-profound sensorineural hearing loss in adults: a systematic review and meta-analysis. Ear Hear. 2016; 37(5):495-507.

Marx M, Costa N, Lepage B, Taoui S, Molinier L, Deguine $\mathrm{O}$, et al. Cochlear implantation as a treatment for single-sided deafness and asymmetric hearing loss: a randomized controlled evaluation of cost-utility. BMC Ear Nose Throat Disord. 2019;19(1):1.

Mertens G, Kleine Punte A, De Bodt M, Van de Heyning P. Binaural auditory outcomes in patients with postlingual profound unilateral hearing loss: 3 years after cochlear implantation. Audiol Neurootol. 2015;20(Suppl 1):6772.

Molinier L, Bocquet H, Bongard V, Fraysse B. The economics of cochlear implant management in France: a multicentre analysis. Eur J Health Econ. 2009; 10:347-55.

Pennings RJ, Gulliver M, Morris DP. The importance of an extended preoperative trial of BAHA in unilateral sensorineural hearing loss: a prospective cohort study. Clin Otolaryngol. 2011;36(5):442-9.

Peters JP, van Zon A, Smit AL, van Zanten GA, de Wit GA, Stegeman I, et al. CINGLE-trial: cochlear implantation for siNGLE-sided deafness, a randomised controlled trial and economic evaluation. BMC Ear Nose Throat Disord. 2015;15:3.

Quaranta N, Wagstaff S, Baguley DM. Tinnitus and cochlear implantation. Int J Audiol. 2004; 43(5):245-51.

Ramakers GG, Smulders YE, van Zon A, Kraaijenga VJ, Stegeman I, Van Zanten GA, et al. Agreement between health utility instruments in cochlear implantation. Clin Otolaryngol. 2016;41(6):737-43.

Saroul N, Akkari M, Pavier Y, Gilain L, Mom T. Baha-mediated rehabilitation of patients with unilateral deafness: selection criteria. Audiol Neurootol. 2014;19(2):85-90.

Slattery WH, Middlebrooks JC. Monaural sound localization: acute versus chronic unilateral impairment. Hear Res. 1994;75(1-2):38-46.

Sullivan CB, Al-Qurayshi Z, Zhu V, Liu A, Dunn C, Gantz BJ, et al. Long-term audiologic outcomes after cochlear implantation for single-sided deafness. Laryngoscope. 2020 Jul;130(7):1805-11.

Távora-Vieira D, De Ceulaer G, Govaerts PJ, Rajan GP. Cochlear implantation improves localization ability in patients with unilateral deafness. Ear Hear. 2015;36(3):e93-8.

Van de Heyning P, Távora-Vieira D, Mertens G, Van Rompaey V, Rajan GP, Müller J, et al. Towards a unified testing framework for single-sided deafness studies: a consensus paper. Audiol Neurootol. 2016;21(6):391-8.

Van de Heyning P, Vermeire K, Diebl M, Nopp P, Anderson I, De Ridder D. Incapacitating unilateral tinnitus in single-sided deafness treated by cochlear implantation. Ann Otol Rhinol Laryngol. 2008;117(9):645-52.

Vannson N, James CJ, Fraysse B, Lescure B, Strelnikov K, Deguine O, et al. Speech-in-noise perception in unilateral hearing loss: relation to pure-tone thresholds and brainstem plasticity. Neuropsychologia. 2017;102:135-43.

Vermeire K, Van de Heyning P. Binaural hearing after cochlear implantation in subjects with unilateral sensorineural deafness and tinnitus. Audiol Neurootol. 2009;14(3):163-71.

Vincent C, Arndt S, Firszt JB, Fraysse B, Kitterick PT, Papsin BC, et al. Identification and evaluation of cochlear implant candidates with asymmetrical hearing loss. Audiol Neurootol. 2015;20(Suppl 1):87-9.

Vlastarakos PV, Nazos K, Tavoulari EF, Nikolopoulos TP. Cochlear implantation for singlesided deafness: the outcomes. An evidencebased approach. Eur Arch Otorhinolaryngol. 2014;271(8):2119-26.

van Zon A, Peters JP, Stegeman I, Smit AL, Grolman W. Cochlear implantation for patients with single-sided deafness or asymmetrical hearing loss: a systematic review of the evidence. Otol Neurotol. 2015;36(2):209-19. 